\title{
Brain opioid-mediated analgesia by systemic administration of dipeptide kyotorphin analog
}

\author{
Hiroyuki Neyama ${ }^{1}$, Yusuke Hamada ${ }^{2}$, Minoru Narita ${ }^{2}$, Hiroshi Ueda $^{1}$ \\ ${ }^{I}$ Department of Pharmacology and Therapeutic Innovation, Nagasaki University, Institute of Biomedical Sciences, \\ Japan, ${ }^{2}$ Department of Pharmacology, Hoshi University School of Pharmacy and Pharmaceutical Sciences, Tokyo, \\ Japan
}

Kyotorphin (L-Tyrosine-L-Arginine) is an opioid-like analgesic dipeptide, which was isolated from bovine brain. Regarding the mechanisms underlying centrally administered kyotorphin-induced analgesia, we have proposed that the met-enkephalin release is one of mechanisms (Nature 1979). The previous studies revealed that kyotorphin binds to putative specific Gi-coupled receptor, and Leucine-Arginine (LR) is a specific antagonist (JBC, 1979). We also found Nmethyl derivatives of kyotorphin (NMYR) and LR (NMLR) are potent and stable agonist and antagonist, respectively (Peptides, 2000). The subcutaneous (s.c.) or per os (p.o.) administration of NMYR causes potent analgesic effects in thermal and mechanical nociception tests in mice. The analgesia was completely blocked by the intracerebroventricular (i. c.v.) injection of NMLR, but slight blocked by the intrathecal (i.t.) injection. Furthermore, the loss of NMYR analgesia was observed in mu opioid receptor (MOPr) KO mice or by the i.c.v. injection of naloxone. These results are consistent to the findings that NMYR analgesia was lost in fibromyalgia-like pain models, which lose central morphine analgesia, but remains in partial sciatic nerve ligation model, which remains central, but not peripheral morphine analgesia. In the in vitro assay, kyotorphin has no positive allosteric agonist activity in $\mathrm{CHO}$ cells expressing MOPr or delta opioid receptor. Finally, NMYR analgesia was partially inhibited in mice deficient of beta-endorphin or met-enkephalin. All these findings support our previous hypothesis that kyotorphin elicits analgesia through a release of endogenous opioids by use of enzymatic stable analog, NMYR. Further molecular mechanisms will be discussed in the presentation. 